\title{
Excitation of nuclear anharmonic vibrations in heavy-ion fusion reactions
}

\author{
K. Hagino ${ }^{1}$, S. Kuyucak ${ }^{2}$, and N. Takigawa ${ }^{1}$ \\ ${ }^{1}$ Department of Physics, Tohoku University, Sendai 980-77, Japan \\ ${ }^{2}$ Department of Theoretical Physics, Research School of Physical Sciences, \\ Australian National University, Canberra, ACT 0200, Australia
}

\begin{abstract}
We discuss the effects of multi-phonon excitations on heavy-ion fusion reactions at energies near and below the Coulomb barrier, focusing especially on the role of anharmonicities. We carry out a systematic study of those effects on the excitation function of the fusion cross section and on the fusion barrier distribution, by using the vibrational limit of the interacting boson model. We also analyze the recently measured high-precision data of the ${ }^{16} \mathrm{O}+{ }^{148} \mathrm{Sm}$ fusion reaction with this model and discuss the anharmonic properties of the quadrupole as well as the octupole vibrations in ${ }^{148} \mathrm{Sm}$. Negative and positive static quadrupole moments are deduced for the first $2^{+}$and $3^{-}$states in ${ }^{148} \mathrm{Sm}$, respectively. It is shown that the fusion barrier distribution strongly depends on the sign of the quadrupole moments, suggesting that subbarrier fusion reactions offer an alternative method to extract the static quadrupole moments of phonon states in spherical nuclei.
\end{abstract}

PACS number(s): 25.70.Jj, 21.60.Fw, 21.10.Ky, 27.60.+j 


\section{INTRODUCTION}

Large enhancement of heavy-ion fusion cross sections at subbarrier energies relative to predictions of a one-dimensional potential model has been well recognized [1]. It is by now well established that these enhancements can be attributed to couplings of the relative motion between the colliding nuclei to their intrinsic excitations as well as transfer reactions [2]. When the excitation energy of an intrinsic motion is neglected, the corresponding channel-coupling gives rise to a distribution of potential barriers [ [3, 因, 可. Based on this idea, a method was proposed to extract barrier distributions directly from fusion excitation functions by taking the second derivative of the product of the fusion cross section and the center of mass energy $E \sigma$ with respect to $E$ [6]. It was recently shown that the concept of the barrier distribution can be extended also to the cases where the intrinsic motion carries a finite excitation energy [7]. Coupled-channels calculations have shown that the fusion barrier distribution, i.e. $d^{2}(E \sigma) / d E^{2}$, is very sensitive to the details of the channel couplings, while the fusion excitation function itself is rather featureless [8]. In order to deduce meaningful experimental barrier distributions and to observe their sensitivity to the couplings, the excitation function of fusion cross sections has to be measured with high precision at small energy intervals. Thanks to the recent developments in experimental techniques [9], such data are now available for several systems, and they have clearly demonstrated that the barrier distribution is indeed a sensitive quantity to the channel couplings [10]. They clearly show the effects of couplings to static deformations and associated rotational motions [10, 11, 12, 13, 14], vibrational degrees of freedom 10, 14, 15, 16, 17, 18], and transfer reactions 110, 14, 15, 16, 17], in a way much more apparent than in the fusion excitation function itself. These high precision data have thus enabled a detailed study of the effects of nuclear collective excitations on fusion reactions, and have generated a renewed interest in heavy-ion subbarrier fusion reactions.

Nuclear surface oscillations with various multipolarities are typical examples where the barrier distribution analyses of fusion reactions may be applied to study their detailed properties. For instance, the barrier distribution analysis of the recently measured accurate data on the ${ }^{58} \mathrm{Ni}+{ }^{60} \mathrm{Ni}$ fusion reaction has shown evidence for couplings to double-phonon states in ${ }^{58} \mathrm{Ni}$ and ${ }^{60} \mathrm{Ni}$ [18]. The barrier distributions were shown to be quite sensitive to the number of phonons excited during fusion reactions. To the zeroth approximation, these surface vibrations can be described in terms of harmonic oscillations, which dictate simple relations among the level energies and the electromagnetic transitions between them. Some of the characteristic features of harmonic oscillations are summarized as follows: (i) all the levels in a phonon multiplet are degenerate and the energy spacing between neighboring multiplets is a constant, (ii) the electric transitions between neighboring multiplets are linearly related, e.g. $\mathrm{B}\left(\mathrm{E} 2 ; \mathrm{I}_{1}^{+} \rightarrow(\mathrm{I}-2)_{1}^{+}\right)=(\mathrm{I} / 2)$ $\mathrm{B}\left(\mathrm{E} 2 ; 2_{1}^{+} \rightarrow 0_{1}^{+}\right)$, (iii) the static quadrupole moment is zero for all the phonon states. In realistic nuclei, however, there are residual interactions between phonons, which cause deviations from the harmonic oscillator limit [19]. The anharmonicity leads to a level splitting within a multiplet and to modifications in the ratios between various electro-

magnetic transition strengths. Furthermore, the anharmonicities generate a finite value of static quadrupole moment in excited states [20]. 
In even-even nuclei near closed shells, there are many examples of two-phonon triplets $\left(0^{+}, 2^{+}, 4^{+}\right)$of quadrupole surface vibrations [21]. Though the central position of their excitation energies is approximately twice the energy of the first $2^{+}$state, they usually exhibit appreciable splitting within the triplet. A theoretical analysis of the anharmonicities for the quadrupole vibrations was first performed by Brink et al. [19, who related the excitation energies of three-phonon quintuplet $\left(0^{+}, 2^{+}, 3^{+}, 4^{+}, 6^{+}\right)$to those of the doublephonon triplets, and also gave relations between the electric transition strengths from the three- to the two-phonon states and those from the two- to the one-phonon states. These relations, however, had not been confirmed until recently because of the sparse experimental data on three-phonon states. The experimental situation has improved rapidly in recent years [22], and data on multi-phonon states are now available for several nuclei. As a consequence, study of multi-phonon states, and especially their anharmonic properties, is attracting much interest [23, 24, 25, 26, 27]. It is worth mentioning that anharmonic effects are not restricted to low-lying vibrations but play significant roles also in multi-phonon excitations of giant resonances in heavy-ion collisions [28, 29, 30].

In many even-even nuclei near closed shells, the first $3^{-}$state appears at a relatively low excitation energy, which competes with the quadrupole state [21, 31]. These low-lying $3^{-}$states have been frequently interpreted as collective octupole vibrations arising from a coherent sum of one-particle one-hole excitations between single particle orbitals differing by three units of orbital angular momentum [21, 32]. This picture is supported by the large E3 transition probabilities from the first $3^{-}$state to the ground state, and suggests the possibility of multi-octupole-phonon excitations. In contrast to the quadrupole vibrations, however, so far there are only little experimental evidence for double-octupole-phonon states. One reason for this is that E3 transitions from two-phonon states to a singlephonon state compete against lower multipolarity E1 transitions. This makes it difficult to unambiguously identify the two-phonon quartet states $\left(0^{+}, 2^{+}, 4^{+}, 6^{+}\right)$. Consequently despite the fact that the first $3^{-}$state of ${ }^{208} \mathrm{~Pb}$ has a large quadrupole moment, which indicates a significant anharmonic effect in octupole vibrations [33, 34, 35, 36], a direct study of the anharmonic properties in multi-octupole phonon spectra has not been possible for a long time. Only in recent years, convincing evidence have been reported for doubleoctupole-phonon states, as well as double-phonon states built from single octupole and quadrupole phonon states, in some nuclei, e.g., ${ }^{208} \mathrm{~Pb}$ [37], ${ }^{144} \mathrm{Sm}$ [38, 39, 40, 41], ${ }^{147} \mathrm{Sm}$ 42], ${ }^{146} \mathrm{Sm}$ 43, ${ }^{145} \mathrm{Nd}$ 42, ${ }^{144} \mathrm{Nd}$ 43, and ${ }^{148} \mathrm{Gd} 44$, 45].

In Ref. [46], we have shown that heavy-ion subbarrier fusion reactions provide a powerful method to study the anharmonic properties of quadrupole as well as octupole vibrational excitations. We analyzed the recently measured high precision data for the ${ }^{16} \mathrm{O}$ $+{ }^{144} \mathrm{Sm}$ reaction and extracted negative quadrupole moments for both the first $2^{+}$and $3^{-}$states in ${ }^{144} \mathrm{Sm}$. The aim of the present paper is, in addition to giving the full details of that analysis, to carry out a systematic study of the effects of anharmonicities on subbarrier fusion, and to apply the same analysis to the ${ }^{16} \mathrm{O}+{ }^{148} \mathrm{Sm}$ fusion reaction to study the anharmonic properties of the vibrational excitations in ${ }^{148} \mathrm{Sm}$ and their effects on the fusion reaction. Although the importance of the anharmonic effects on proton scattering [47, as well as the reorientation effects in phonon spectra on heavy-ion elastic and inelastic scattering [48, 49] has been pointed out, no systematic studies of the effects 
of anharmonicities on subbarrier fusion and on fusion barrier distributions have been performed so far. In view of the high precision data on subbarrier fusion reactions, which have recently become available, such studies are necessary in discussing the effects of vibrational excitations on subbarrier fusion reactions. The paper is organized as follows. In Sec. II, we formulate coupled-channels calculations which explicitly take into account the anharmonic properties of vibrational excitations using the vibrational limit of the interacting boson model [50]. We compare the formalism with that in the harmonic limit and point out several important features of the anharmonic vibrational excitations. In Sec. III, a systematic study of the effects of anharmonicities on fusion reactions is presented. They include the effects of unequal spacing of levels, reorientation, and finite boson number. In Sec. IV, the formalism is applied to the ${ }^{16} \mathrm{O}+{ }^{148} \mathrm{Sm}$ fusion reaction. We extract the quadrupole moments of the first $2^{+}$and $3^{-}$states in ${ }^{148} \mathrm{Sm}$ from the analysis of the high quality fusion data available for this system. Finally, a summary of the work is given in Sec. V.

\section{EFFECTS OF PHONON EXCITATIONS ON FUSION}

In this section, we describe the basic formalism for treating the vibrational excitation in subbarrier fusion reactions. Let us first consider the case where the relative motion between the colliding nuclei couples to the quadrupole vibrations in the target nucleus. The total Hamiltonian of the system is assumed to be

$$
H=-\frac{\hbar^{2}}{2 \mu} \nabla^{2}+V_{N}(r)+\frac{Z_{P} Z_{T} e^{2}}{r}+H_{\text {int }}+V_{\text {coup }}(\boldsymbol{r}, \xi),
$$

where $\mathbf{r}$ is the coordinate of the relative motion between the projectile and the target, and $\mu$ is the reduced mass. $V_{N}$ is the bare nuclear potential, $Z_{P}$ and $Z_{T}$ are the atomic numbers of the projectile and the target, respectively. $H_{\text {int }}$ describes the vibrational excitations in the target nucleus, and $V_{\text {coup }}$, the coupling between these excitations (generically denoted by $\xi$ ) and the relative motion. In Sub-sections A and B, we discuss the coupling of the harmonic and anharmonic vibrators within the linear coupling approximation. Although this approximation is too simplistic to apply to realistic systems [51, 22, it enables us to easily understand the essential features of the effects of anharmonicities. Extensions of the model so as to include the couplings to all orders and to the octupole vibrations in the target nucleus as well are given in Sub-sections C and D, respectively.

\section{A. Harmonic limit}

In the geometrical model of Bohr and Mottelson, the radius of the vibrating target nucleus is parameterized as

$$
R(\theta, \phi)=R_{T}\left(1+\sum_{\mu} \alpha_{2 \mu} Y_{2 \mu}^{*}(\theta, \phi)\right),
$$

where $R_{T}$ is the equivalent sharp surface radius of the target nucleus and $\alpha_{2 \mu}$ are the coordinates of the surface vibration. To the lowest order, the surface oscillations are 
approximated by a harmonic oscillator and the corresponding Hamiltonian is given by

$$
H_{\text {int }}=\hbar \omega\left(\sum_{\mu} a_{2 \mu}^{\dagger} a_{2 \mu}+\frac{5}{2}\right) \text {. }
$$

Here $\hbar \omega$ is the oscillator quanta and $a_{2 \mu}^{\dagger}$ and $a_{2 \mu}$ are the phonon creation and annihilation operators, respectively. The surface coordinates $\alpha_{2 \mu}$ and the phonon creation and annihilation operators are related by

$$
\alpha_{2 \mu}=\alpha_{0}\left(a_{2 \mu}^{\dagger}+(-)^{\mu} a_{2 \mu}\right),
$$

where $\alpha_{0}$ is the amplitude of the zero point motion. It is related to the quadrupole deformation parameter by $\alpha_{0}=\beta_{2} / \sqrt{5}$ [21] and can be estimated from the experimental transition probability using

$$
\alpha_{0}=\frac{1}{\sqrt{5}} \frac{4 \pi}{3 Z_{T} R_{T}^{2}} \sqrt{\frac{B(E 2) \uparrow}{e^{2}}} .
$$

In the collective model, the coupling Hamiltonian between the relative motion and the quadrupole surface oscillations is often assumed to be

$$
V_{\text {coup }}\left(\mathbf{r}, \alpha_{2}\right)=f(r) \sum_{\mu} \alpha_{2 \mu} Y_{2 \mu}^{*}(\hat{\mathbf{r}}) .
$$

This is the so called linear coupling approximation where only the linear term with respect to the surface coordinate is taken into account. The coupling form factor, $f(r)$, consists of the nuclear and the Coulomb parts and is given by

$$
f(r)=-R_{T} \frac{d V_{N}}{d r}+\frac{3}{5} Z_{P} Z_{T} e^{2} \frac{R_{T}^{2}}{r^{3}}
$$

The dimension of the resulting coupled-channels calculations is in general too large for practical purposes. Here, we introduce two approximations which considerably reduce the dimension of the coupled-channels calculations with negligible effects on its accuracy. The first one is the no-Coriolis approximation where one transforms the whole system to the rotating frame where the $z$ axis is along the direction of the relative motion $\mathbf{r}$ at every instant [53, 54]. This is achieved by replacing the angular momentum of the relative motion in each channel by the total angular momentum $J$. As the operator for a rotational coordinate transformation in the whole space then commutes with the centrifugal operator for the relative motion, one can make this transformation to the rotating frame without introducing any complications [54. Using $Y_{2 \mu}(\hat{\mathbf{r}}=0)=\sqrt{5 / 4 \pi} \delta_{\mu, 0}$, the coupling Hamiltonian (6) in the rotating frame becomes

$$
V_{\text {coup }}\left(\mathbf{r}, \alpha_{2}\right)=\sqrt{\frac{5}{4 \pi}} f(r) \alpha_{20}=\frac{\beta_{2}}{\sqrt{4 \pi}} f(r)\left(a_{20}^{\dagger}+a_{20}\right) .
$$

Since the projection of the total angular momentum $J$ onto the $z$-axis of the rotating frame, i.e. the $K$ quantum number, is conserved in this approximation, the dimension of the coupled-channels equations is drastically reduced. 
A further reduction of the coupled-channels equations can be achieved by introducing the $n$-phonon channels [53, 55]. For the quadrupole surface vibrations, the two phonon state has three levels $\left(0^{+}, 2^{+}, 4^{+}\right)$. In the harmonic limit, this two-phonon triplet is degenerate in the excitation energy. One can then replace the couplings to all the members of the two-phonon triplet by the coupling to a single state given by

$$
\left|2>=\sum_{I=0,2,4}<2020\right| I 0>\left|I 0>=\frac{1}{\sqrt{2 !}}\left(a_{20}^{\dagger}\right)^{2}\right| 0>.
$$

In the same way, one can introduce the $n$-phonon channel as

$$
\left|n>=\frac{1}{\sqrt{n !}}\left(a_{20}^{\dagger}\right)^{n}\right| 0>.
$$

If we truncate to the two-phonon states, the corresponding coupling matrix is given by

$$
V_{\text {coup }}=\left(\begin{array}{ccc}
0 & F(r) & 0 \\
F(r) & \hbar \omega & \sqrt{2} F(r) \\
0 & \sqrt{2} F(r) & 2 \hbar \omega
\end{array}\right),
$$

where, $F(r)$ is defined as $\beta_{2} f(r) / \sqrt{4 \pi}$.

\section{B. Anharmonic vibrator}

Higher order corrections to Eq. (3) lead to anharmonicities in the vibrational excitation. The effects of anharmonicity in surface vibrations can be described in many different ways. Among them, the interacting boson model (IBM) [50] in the vibrational (U(5)) limit provides a convenient calculational framework to discuss the anharmonic effects. The vibrational limit of the IBM and the anharmonic vibrator (AHV) in the geometrical model are very similar, the only difference coming from the finite number of bosons in the former [22, 56, 57]. The eigenvalues of the intrinsic Hamiltonian $H_{\text {int }}$ in the U(5) limit are given by [50]

$$
E_{n_{d} v I}=\epsilon n_{d}+\alpha n_{d}\left(n_{d}+4\right)+\beta v(v+3)+\gamma I(I+1),
$$

where $n_{d}, v$, and $I$ are the quantum numbers giving the number of $d$-bosons, the $d$-boson seniority, and the intrinsic angular momentum, respectively. $\epsilon, \alpha, \beta$, and $\gamma$ are adjustable parameters. The first term gives equally spaced and degenerate phonon spectra, while the splitting of multi-phonon multiplets due to the anharmonic effects are caused by the remaining terms.

A model for subbarrier fusion reactions, which uses the IBM to describe the effects of channel couplings, has been developed by Balantekin et al. [51, 58, 59]. They assume that the coupling Hamiltonian has a similar form to that of the collective model Eq. (6). In the linear coupling and the no-Coriolis approximations, it is given as

$$
V_{\text {coup }}(r, \xi)=\frac{\beta_{2}}{\sqrt{4 \pi N}} f(r) Q_{20} .
$$


Here, $N$ is the boson number and we have introduced the scaling of the coupling strength with $\sqrt{N}$ to ensure the equivalence of the IBM and the geometric model results in the large $N$ limit [51, 58, 59]. $Q_{20}$ is the quadrupole operator in the IBM, which is given by

$$
Q_{20}=s^{\dagger} d_{0}+s d_{0}^{\dagger}+\chi_{2}\left(d^{\dagger} \tilde{d}\right)_{0}^{(2)},
$$

where tilde is defined as $\tilde{d}_{\mu}=(-)^{\mu} d_{-\mu}$. Systematic studies of subbarrier fusion reactions in this model indicate that the coupling strengths used in this model are very similar to those in the geometrical model [58]. We, therefore, assume that the coupling strength in Eq. (13) is the same as that in the harmonic limit of the geometrical model, Eq. (8). This approximation is valid when the anharmonic effects are not so large, so as to allow description of vibrational mode of excitations in terms of the U(5) limit.

In anharmonic vibrators, phonon states within a given multiplet are split in energy. Thus one has to treat each state separately as a different channel. The introduction of the multi-phonon channel, as in the harmonic limit, is possible only when the multi-phonon states are degenerate in the excitation energies. It will be shown in the next section that one can safely neglect the effects of this energy splitting on fusion cross section as well as on the fusion barrier distribution. Accordingly, in order to keep the calculations simple, we assume in the following that the multi-phonon multiplets are degenerate in their excitation energy unless explicitly mentioned. The wave function of the $n$-phonon channel in the framework of the IBM then reads

$$
\left|n>=\frac{1}{\sqrt{n !(N-n) !}}\left(s^{\dagger}\right)^{N-n}\left(d_{0}^{\dagger}\right)^{n}\right| 0>,
$$

and the corresponding coupling matrix, truncated to the two-phonon states, is given by

$$
V_{\text {coup }}=\left(\begin{array}{ccc}
0 & F(r) & 0 \\
F(r) & \hbar \omega-\frac{2}{\sqrt{14 N}} \chi_{2} F(r) & \sqrt{2(1-1 / N)} F(r) \\
0 & \sqrt{2(1-1 / N)} F(r) & 2 \hbar \omega+\delta-\frac{4}{\sqrt{14 N}} \chi_{2} F(r)
\end{array}\right) .
$$

The parameter $\delta$ has been introduced to represent deviation of the energy spectrum from the equal spacing in the harmonic oscillator limit. When the $\chi_{2}$ parameter in the quadrupole operator is zero, quadrupole moments of all states vanish, and one obtains the harmonic limit in the large $N$ limit. Non-zero values of $\chi_{2}$ generate quadrupole moments and, together with finite boson number, are responsible for the anharmonicities in electric transitions. The differences in the coupling scheme in the harmonic oscillator limit and in the case with anharmonicities are summarized schematically in Fig. 1.

\section{All order couplings}

It has been pointed out that the linear coupling approximation for the nuclear coupling is not adequate in heavy-ion fusion reactions [51, 52]. Higher order couplings have to be included in order to describe realistic systems and compare the theoretical calculations with the experimental data. On the other hand, non-linear terms were shown to be 
negligible for the Coulomb couplings [52]. Higher order corrections to the nuclear coupling were discussed in Ref. [52] in the case of a harmonic oscillator. The coupling matrix element between $n$ - and $m$ - phonon channels in the harmonic limit is given by

$$
V_{n m}^{(N)}(r)=\int_{-\infty}^{\infty} d x u_{n}^{*}(x) u_{m}(x) \frac{-V_{0}}{1+\exp \left[\left(r-R_{0}-\sqrt{\frac{5}{4 \pi}} R_{T} x\right) / a\right]}
$$

Here we have adopted the no-Coriolis approximation and the nuclear potential is assumed to have a Woods-Saxon form. Note that this equation includes the bare nuclear potential $V_{N}$ in the total Hamiltonian (Eq. (1)). $u_{n}(x)$ is the wavefunction of the $n$-th excited state of the harmonic oscillator (see Eq. (10)) and is given by

$$
u_{n}(x)=\frac{1}{\sqrt{2^{n} n !}} \frac{1}{\sqrt[4]{2 \pi \alpha_{0}^{2}}} H_{n}\left(x / \sqrt{2} \alpha_{0}\right) e^{-x^{2} / 4 \alpha_{0}^{2}},
$$

where $H_{n}(x)$ is the Hermite polynomial.

Extension of the anharmonic oscillator coupling in the linear order approximation to all orders can be carried out in a similar manner. Following Refs. [51, 58, 59], we assume that the nuclear coupling Hamiltonian is given in the no-Coriolis approximation by

$$
V^{(N)}(r)=\frac{-V_{0}}{1+\exp \left[\left(r-R_{0}-R_{T} \frac{\beta_{2}}{\sqrt{4 \pi N}} Q_{20}\right) / a\right]} .
$$

The matrix elements of Eq. (19) can be evaluated most easily by introducing the interaction representation which diagonalizes the quadrupole operator $Q_{20}$ [59]. Since phonon states $|n, I, M\rangle$ with $M \neq 0$ do not couple to the ground state in the no-Coriolis approximation, we do not need to consider the terms $d_{m}^{\dagger} d_{m}$ with $m \neq 0$ in the quadrupole operator, Eq. (14). Accordingly, we diagonalize the operator

$$
Q_{20}=s^{\dagger} d_{0}+s d_{0}^{\dagger}+c_{0} d_{0}^{\dagger} d_{0}
$$

where $c_{0}=-\sqrt{2 / 7} \chi_{2}$, in the interaction basis as

$$
Q_{20}=e_{+} B_{+}^{\dagger} B_{+}+e_{-} B_{-}^{\dagger} B_{-},
$$

where $B_{ \pm}^{\dagger}\left(B_{ \pm}\right)$are creation (annihilation) operators of the eigenbosons and $e_{ \pm}$are their eigenvalues. After working out the commutators

$$
\left[Q_{20}, B_{ \pm}^{\dagger}\right]=e_{ \pm} B_{ \pm}^{\dagger}
$$

we find that they are given by

$$
\begin{aligned}
& B_{+}^{\dagger}=y_{0} s^{\dagger}+y_{2} d_{0}^{\dagger}, \\
& B_{-}^{\dagger}=-y_{2} s^{\dagger}+y_{0} d_{0}^{\dagger}, \\
& e_{ \pm}=\frac{c_{0} \pm \sqrt{4+c_{0}^{2}}}{2},
\end{aligned}
$$


with $y_{0}$ and $y_{2}$ are defined as

$$
\begin{aligned}
& y_{0}=\frac{1}{\sqrt{1+e_{+}^{2}}}=\sqrt{\frac{\sqrt{4+c_{0}^{2}}-c_{0}}{2 \sqrt{4+c_{0}^{2}}}}, \\
& y_{2}=\frac{e_{+}}{\sqrt{1+e_{+}^{2}}}=\sqrt{\frac{\sqrt{4+c_{0}^{2}}+c_{0}}{2 \sqrt{4+c_{0}^{2}}}} .
\end{aligned}
$$

The eigenvectors of the quadrupole operator are then given by

$$
\left|n_{+}, n_{-}>=\frac{1}{\sqrt{n_{+} ! n_{-} !}}\left(B_{+}^{\dagger}\right)^{n_{+}}\left(B_{-}^{\dagger}\right)^{n_{-}}\right| 0>
$$

and the matrix elements of the nuclear coupling Hamiltonian between $n$ - and $m$ - phonon channels read

$$
\begin{aligned}
V_{n m}^{(N)}(r)=\quad & \sum_{k=0}^{N} \frac{-V_{0}}{1+\exp \left[\left(r-R_{0}-R_{T} \frac{\beta_{2}}{\sqrt{4 \pi N}}\left(k e_{+}+(N-k) e_{-}\right)\right) / a\right]} \\
& \times \sum_{i, j=0}^{k}(-)^{n+m+i+j}\left(\begin{array}{c}
k \\
i
\end{array}\right)\left(\begin{array}{c}
N-k \\
N-n-i
\end{array}\right)\left(\begin{array}{c}
k \\
j
\end{array}\right)\left(\begin{array}{c}
N-k \\
N-m-j
\end{array}\right) \\
& \times \frac{\sqrt{(N-n) ! n !(N-m) ! m !}}{k !(N-k) !} y_{0}^{2 i+2 j-2 k+m+n} y_{2}^{2 k+2 N-m-n-2 i-2 j} .
\end{aligned}
$$

In evaluating Eq. (29), we used the fact that the sum of the number of each eigenfunction bosons, $n_{+}+n_{-}$, must be equal to the total boson number $N$.

\section{Coupling to octupole mode}

In spherical nuclei, the low-lying octupole vibrational state has a large collectivity and their excitations play an important role in subbarrier fusion reactions. Therefore, in order to apply the models which we discussed above to realistic systems, it is necessary to extend them so that they include the octupole mode as well. The coupling Hamiltonian in the harmonic limit can be extended in a straightforward way and reads

$$
V^{(N)}(r, \alpha)=\frac{-V_{0}}{1+\exp \left[\left(r-R_{0}-\sqrt{\frac{5}{4 \pi}} R_{T} \alpha_{20}-\sqrt{\frac{7}{4 \pi}} R_{T} \alpha_{30}\right) / a\right]},
$$

and

$$
V^{(C)}(r, \alpha)=\frac{3}{5} Z_{P} Z_{T} e^{2} \frac{R_{T}^{2}}{r^{3}} \sqrt{\frac{5}{4 \pi}} \alpha_{20}+\frac{3}{7} Z_{P} Z_{T} e^{2} \frac{R_{T}^{3}}{r^{4}} \sqrt{\frac{7}{4 \pi}} \alpha_{30},
$$

for the nuclear and the Coulomb couplings, respectively. Here $\alpha_{20}$ and $\alpha_{30}$ are the surface

coordinates for the quadrupole and the octupole vibrations, respectively. In order to take into account the octupole mode of excitation in the anharmonic oscillator coupling, we use the vibrational limit of the $s d f$ - IBM [50, 60]. This model has been successfully 
used to describe negative parity states in rotational [60] as well as vibrational 66, 62, 63] nuclei. As a natural extension, we assume the following coupling Hamiltonians based on this model for the nuclear and the Coulomb couplings.

$$
\begin{aligned}
V^{(N)}(r, \xi) & =\frac{-V_{0}}{1+\exp \left[\left(r-R_{0}-R_{T} \frac{\beta_{2}}{\sqrt{4 \pi N}} Q_{20}-R_{T} \frac{\beta_{3}}{\sqrt{4 \pi N}} Q_{30}\right) / a\right]}, \\
V^{(C)}(r, \xi) & =\frac{3}{5} Z_{P} Z_{T} e^{2} \frac{R_{T}^{2}}{r^{3}} \frac{\beta_{2}}{\sqrt{4 \pi N}} Q_{20}+\frac{3}{7} Z_{P} Z_{T} e^{2} \frac{R_{T}^{3}}{r^{4}} \frac{\beta_{3}}{\sqrt{4 \pi N}} Q_{30} .
\end{aligned}
$$

Here, $\beta_{3}$ is the octupole deformation parameter, and we take the quadrupole and the octupole operators in the $s d f$ - IBM as

$$
\begin{aligned}
& \hat{Q}_{2}=s^{\dagger} \tilde{d}+s d^{\dagger}+\chi_{2}\left(d^{\dagger} \tilde{d}\right)^{(2)}+\chi_{2 f}\left(f^{\dagger} \tilde{f}\right)^{(2)}, \\
& \hat{Q}_{3}=s f^{\dagger}+\chi_{3}\left(\tilde{d} f^{\dagger}\right)^{(3)}+h . c .
\end{aligned}
$$

respectively, where $\tilde{f}_{\mu}$ is defined as $(-)^{3+\mu} f_{-\mu}$. We will apply these models in Sec. IV to analyze the ${ }^{16} \mathrm{O}+{ }^{148} \mathrm{Sm}$ fusion reaction.

\section{ANHARMONIC EFFECTS ON FUSION BARRIER DISTRIBUTIONS}

In order to discuss the effects of anharmonicity on subbarrier fusion reactions, we perform in this section a series of model calculations without particular regard for the physical values of the parameters. We consider a fictitious nucleus whose excitation energies are given by Eq. (12) with $\epsilon=800, \alpha=10, \beta=0$ and $\gamma=25$, all in $\mathrm{keV}$. The excitation energy of the first $2^{+}$state of this nucleus is $1 \mathrm{MeV}$ and the double phonon states are split by $0.5 \mathrm{MeV}$. We take the total boson number $N$ to be 4 and thus take into account coupling of up to four phonon states. The other parameters used are $\chi_{2}=-3$ (see Eq. (14)), the quadrupole deformation parameter $\beta_{2}=0.25$ and the target radius $R_{T}=6.35 \mathrm{fm}$. Finally, the Christensen-Winther parameterization of the Woods-Saxon potential [64] for the ${ }^{16} \mathrm{O}+{ }^{148} \mathrm{Sm}$ system is used for the ion-ion potential $V_{N}$.

As we discussed in the previous section, introduction of the multi-phonon channels significantly reduces the dimension of the coupled-channels equations. To see whether this approximation is good, a calculation is first made by taking fully into account the effects of the splittings in the phonon spectrum. In order to keep the calculation simple, we make here the linear coupling approximation. In Fig. 2, we show the excitation function of the fusion cross section (the upper panel) and the barrier distribution (the lower panel) that follow from using the U(5) limit as described above (solid line). Barrier distributions are obtained using a point-difference formula [10] with $\Delta E=2 \mathrm{MeV}$ in the laboratory frame. To see the effect of the splittings of the multi-phonon states, we have repeated the same calculation by assuming that the $n$-phonon multiplets are degenerate at the excitation energy of the first $(2 n)^{+}$state. We found that this prescription leads to no visible change in the fusion barrier distribution compared to the actual case with splitt energy levels. In the following, we shall therefore assume a degenerate multi-phonon spectrum and use a single multi-phonon channel representing all the states in a multiplet. 
In Fig. 2, we also compare the results for the anharmonic vibrator with those in the harmonic limit (the dotted line). One can observe that the anharmonic effects in the vibrational excitations lead to a significant change in the barrier distribution, though the excitation function of the fusion cross section itself is modified only marginally.

\section{A. Anharmonicities in excitation energies}

In all the following calculations, we take into account the couplings to all orders. We first discuss the effects of the deviation of the energy spectrum from the harmonic limit. In order to isolate these effects, we truncate the phonon spectrum at the double-phonon states in this subsection. Fig. 3 shows the dependence of the fusion cross section (the upper panel) and the fusion barrier distribution (the lower panel) on the parameter $\delta$ in Eq. (16). The cross section and the barrier distribution are calculated for three different values of $\delta$. The solid line is the result when $\delta=0$, while the dotted and the dashed lines are obtained by setting $\delta$ to be 0.5 and $-0.5 \mathrm{MeV}$, respectively. Despite the fact that an unrealistically large value is used for $\delta$ so as to maximize its effect, the barrier distribution changes only slightly for different choices of $\delta$. This indicates that the main effects of anharmonicity on fusion barrier distributions come from the deviation of the transition probabilities from the harmonic limit, including the reorientation effect, rather than anharmonicities in the level energies. Note that this observation does not necessarily mean that fusion reactions are not sensitive to the excitation energy of the phonon states. To demonstrate this, we show in Fig. 3 also the result when both $\hbar \omega$ and $\delta$ is set to be zero (the dot-dashed line). One can see sizable effects of the finite excitation energy of the phonon states on the fusion barrier distribution as well as fusion cross section. We thus conclude that, though the fusion cross section and the barrier distribution are sensitive to the energy of the single-phonon state, they do not depend so much on the excitation energies of the multi-phonon states once the phonon quanta $\hbar \omega$ is fixed.

\section{B. Reorientation effects}

One of the pronounced features of an anharmonic vibrator is that the excited states have non-zero quadrupole moments [20, 21]. In the IBM, the E2 operator is defined as $T(\mathrm{E} 2)=e_{B} \hat{Q}_{2}$, where the quadrupole operator was introduced in Eqs. (14) and (34) for the $s d$ - and $s d f$-IBM, respectively, $e_{B}$ being is the effective charge. In the U(5) limit, the E2 effective charge $e_{B}$ is related to the E2 transition probability by

$$
B\left(E 2 ; 0_{1}^{+} \rightarrow 2_{1}^{+}\right)=5 e_{B}^{2} N .
$$

Using the definition of the static quadrupole moment of a state with spin $I$

$$
Q(I)=\sqrt{\frac{16 \pi}{5}}<I I|T(E 2)| I I>,
$$

we obtain for the quadrupole moment of the $2^{+}$state

$$
Q\left(2^{+}\right)=\sqrt{\frac{32 \pi}{35}} e_{B} \chi_{2} .
$$


The corresponding formula for the first $3^{-}$state reads

$$
Q\left(3^{-}\right)=\sqrt{\frac{20 \pi}{21}} e_{B} \chi_{2 f} .
$$

Fig. 4 shows the influence of the sign of the quadrupole moment of the first $2^{+}$state on the fusion cross section (the upper panel) and the fusion barrier distribution (the lower panel). The solid line corresponds to the negative static quadrupole moment, while the dotted line is obtained by inverting the sign of the $\chi_{2}$ parameter in Eq. (14). As seen from Eq. (38), this is equivalent to taking the opposite sign for the quadrupole moment of the excited state. The dashed line is the result when $\chi_{2}=0$. Fig. 4 demonstrates that the fusion cross section, and especially fusion barrier distribution, strongly depend on the sign of the $\chi_{2}$ parameter, hence on that of the static quadrupole moment. This fact will be used in the next section to determine the quadrupole moments of the first $2^{+}$ and $3^{-}$states in ${ }^{148} \mathrm{Sm}$ from the experimental fusion barrier distribution for the ${ }^{16} \mathrm{O}+$ ${ }^{148} \mathrm{Sm}$ reaction.

\section{Finite $N$ effects}

The other important effect of the deviation of the electric transition rates from the harmonic limit is caused by the finiteness of the boson number. Eq. (16) and Fig. 1 indicate that the anharmonic effects weaken the coupling between the one- and two-phonon states by the factor $\sqrt{1-1 / N}$ compared with that in the harmonic limit. For small values of the boson number $N$, this factor is significantly smaller than one and large anharmonic effects on fusion reactions are expected. In order to demonstrate the finite boson number effects, we show in Fig. 4 also the results in the harmonic limit by the dot-dashed line. One can see a significant difference between this result and that for the zero quadrupole moment (the dashed line), indicating the importance of the finite boson number effect.

Finite boson number effects can also be studied in another, perhaps more instructive way. Since the couplings to the multi-phonon states are weaker than those in the harmonic limit if the anharmonic effects are present, one expects that fusion excitation function converges more rapidly compared with the case in the absence of the anharmonic effects. Fig. 5 shows how the fusion cross sections (the upper panel) and the fusion barrier distributions (the lower panel) converge with the phonon number for fixed total boson number $N=4$. Although the fusion cross section and the fusion barrier distribution obtained by including only the single-phonon excitations are quite different from those in the double-phonon couplings, the difference between the two- and the three-phonon couplings are small. We observe that the fusion barrier distribution almost converges at the three-phonon level. The corresponding calculations in the harmonic limit are performed for comparison. These results are shown in Fig. 6. Contrary to the results for the anharmonic vibrator, the barrier distribution obtained by taking into account up to the four-phonon excitations is still significantly different from that obtained by including up to the three-phonon excitations. We thus conclude that the finite boson number significantly influences the role of higher phonon states in determining the fusion barrier distribution. 


\section{IV. ${ }^{16} \mathrm{O}+{ }^{148} \mathrm{Sm}$ REACTION: COMPARISON WITH EXPERIMENTAL DATA}

We now apply the formalism described in Sec. II to a realistic system. We analyze in particular the ${ }^{16} \mathrm{O}+{ }^{148} \mathrm{Sm}$ reaction, whose excitation function of the fusion cross section was recently measured with high accuracy [10]. We take both the quadrupole and the octupole vibrational excitations in ${ }^{148} \mathrm{Sm}$ into account, while the excitations of ${ }^{16} \mathrm{O}$ are not explicitly included in the coupled-channels calculations. The latter have been shown to lead only to the shift of the fusion barrier distribution in energies without significantly altering its shape [65] and hence can be incorporated in the choice of the bare potential.

In order to qualitatively understand the effects of the channel couplings on this reaction, calculations are first performed by assuming the harmonic limit. The quadrupole and the octupole deformation parameters are estimated from the experimental electric transition probabilities between the ground and the one-phonon states [66 to be $\beta_{2}=0.182$ and $\beta_{3}=0.236$, respectively, by assuming the target radius of $R_{T}=1.06 A^{1 / 3} \mathrm{fm}$. The excitation energies of the phonon states in ${ }^{148} \mathrm{Sm}$ are 0.55 and $1.16 \mathrm{MeV}$, for the first $2^{+}$ and $3^{-}$states, respectively. The parameters for the bare ion-ion potential are obtained by fitting the experimental fusion cross sections. It has been pointed out that the effects of channel couplings play an important role in determining the bare potential for the ${ }^{16} \mathrm{O}+{ }^{144} \mathrm{Sm}$ reaction [67. Accordingly, we fit the experimental data by assuming the three phonon couplings in the harmonic limit (see the following discussion). We use the experimental data between $200 \mathrm{mb}$ and $400 \mathrm{mb}$ to determine the bare potential. We choose this range because, more details of the channel couplings would be important in the lower energy region, while some other effects, e.g. the angular momentum truncation or the dissipation mechanism might play some role [68] in the higher energy region. The best fit parameters which we obtain are $V_{0}=155.1 \mathrm{MeV}, R_{0}=0.95\left(A_{P}^{1 / 3}+A_{T}^{1 / 3}\right) \mathrm{fm}$, and $a=1.05 \mathrm{fm}$, for the depth, the radius, and the diffuseness parameters of the Woods-Saxon potential, respectively. Note that the experimental data at high energy region still require a large surface diffuseness parameter [10] even after including the effects of the channel couplings. The origin of this is not fully understood.

The results for the fusion barrier distribution for the ${ }^{16} \mathrm{O}+{ }^{148} \mathrm{Sm}$ reaction are shown in Fig. 7. The panels differ from each other by the number of the quadrupole phonons coupled. The experimental data are taken from ref. [10]. The point difference formula with $\Delta E=2 \mathrm{MeV}$ in the laboratory frame is used to obtain the fusion barrier distribution. Calculations, which assume that the octupole vibration in ${ }^{148} \mathrm{Sm}$ has only a single-phonon state, are indicated by the dotted lines in Fig. 7. In the three phonon calculation, for example, we included the $2^{+}, 3^{-}, 2^{+} \otimes 2^{+}, 2^{+} \otimes 3^{-}, 2^{+} \otimes 2^{+} \otimes 2^{+}$, and $2^{+} \otimes 2^{+} \otimes 3^{-}$states in ${ }^{148} \mathrm{Sm}$ in the harmonic limit. The coupling scheme in the other panels is defined in the same way. All the dotted lines which are obtained by assuming the single-octupole-phonon excitations in ${ }^{148} \mathrm{Sm}$ do not account for the experimental fusion barrier distribution. However, double-phonon excitations have been found in the neighbouring nuclei, i.e. ${ }^{144} \mathrm{Sm}$ 38, 39, 40, 41], ${ }^{146} \mathrm{Sm} \mathrm{[43],} \mathrm{and}{ }^{147} \mathrm{Sm}$ [42]. We therefore repeat the same calculations by assuming the double-octupole excitations in ${ }^{148} \mathrm{Sm}$ (the solid lines). One can now observe that the experimental data can be explained reasonably well by the three-phonon calcu- 
lations. The fit is not as good if the four phonon quadrupole excitations are included in the coupled channels analysis. These calculations in the harmonic limit thus suggest that there are strong couplings up to the three quadrupole phonon states in ${ }^{148} \mathrm{Sm}$ and the coupling between the three and four phonon states are not as strong as that expected from the harmonic oscillator coupling. They also suggest that there might be double-octupole phonon excitations in ${ }^{148} \mathrm{Sm}$.

Calculations which take into account the anharmonicities of the vibrational excitations are performed next. Following the results of the analysis in the harmonic oscillator limit, we include the octupole excitations up to the double phonon levels and take the total boson number $N=4$. The latter is consistent with the existence of the $Z=64$ subshell closure [56]. The standard prescription for the boson number (i.e. counting pairs of nucleons above or below the nearest shell closure) would give $N=8$ for ${ }^{148} \mathrm{Sm}$. However, it is well known that due to the $Z=64$ subshell closure, the effective boson number is much smaller. The suggested effective number in the literature for the proton bosons varies between $N_{\pi}=1$ and 3 [69, 70], leading to the total boson number to be between 3 and 5 for ${ }^{148} \mathrm{Sm}$. There are three other parameters, i.e. $\chi_{2}, \chi_{2 f}$, and $\chi_{3}$ parameters in the transition operators Eqs. (34) and (35), which need to be determined. Two of them, $\chi_{2}$ and $\chi_{2 f}$, are directly related to the quadrupole moment of the phonon states, and the other the coupling between the quadrupole and the octupole modes. Though there exist experimental data on the quadrupole moment of the first $2^{+}$state of ${ }^{148} \mathrm{Sm}$ [66], the experimental uncertainty is still large. There are no other experimental data which can be used to fix the $\chi_{2 f}$ and $\chi_{3}$ parameters. Therefore, a $\chi^{2}$ fit to the experimental fusion cross sections is carried to determine all the three parameters. In the fitting procedure, all the experimental data below $200 \mathrm{mb}$ are used except for the one at $\mathrm{E}_{l a b}=69.36 \mathrm{MeV}$, which lies outside the systematics. The best fit values are $\chi_{2}=-3.12 \pm 0.77, \chi_{2 f}=4.63 \pm 0.43$, and $\chi_{3}=-1.99 \pm 0.26$. The solid line in Fig. 8 shows the fusion cross section (the upper panel) and the fusion barrier distribution (the lower panel) thus obtained. Following the discussion in the previous section, harmonic spectra for the excitation energies of the phonon states are assumed. One observes that the shape of the fusion barrier distribution obtained by including up to four phonon states with anharmonicities is very similar to that for the three phonon couplings in the harmonic limit (see Fig. 7) and that they agree quite well with the experimental data. This situation is similar to that observed in ref. [46], where it has been shown that the shape of the fusion barrier distribution for the ${ }^{16} \mathrm{O}$ $+{ }^{144} \mathrm{Sm}$ reaction obtained by including up to single phonon states in the harmonic limit is very similar to that for the double phonon couplings with anharmonicities.

In the last section, we discussed two main effects of the anharmonicities in the electric transitions, i.e. the reorientation and the finite $N$ effects on subbarrier fusion reactions. In order to see each effect separately, we repeat the same calculation by setting both the $\chi_{2}$ and $\chi_{2 f}$ parameters to be zero. As was discussed in the previous section, this prescription is equivalent to taking only the finite $N$ effects into account. The result is shown by the dashed line in Fig. 8. The figure also shows for comparison the result obtained by assuming that there are four quadrupole phonon states in the harmonic oscillator limit in ${ }^{148} \mathrm{Sm}$ (the dotted line). The difference between the dotted and the dashed lines, and also between the dashed and the solid lines, is significant. This suggests that both the 
reorientation and the finite $N$ effects play an important role in the fusion reaction.

From the $\chi_{2}$ and $\chi_{2 f}$ parameters which are obtained by the $\chi^{2}$ fit to the experimental fusion cross sections, we estimate the quadrupole moments of the first $2^{+}$and $3^{-}$states of ${ }^{148} \mathrm{Sm}$. The E2 effective charge $e_{B}$ is estimated to be 0.19 eb from the experimental $B(E 2)$ value (see Eq. (36)). Using Eqs. (38) and (39), they are estimated to be $Q\left(2^{+}\right)=$ $-1.00 \pm 0.25 b$ and $Q\left(3^{-}\right)=+1.52 \pm 0.14 b$, respectively. The quadrupole moment of the first $2^{+}$state thus obtained is very close to that measured from the Coulomb excitation technique, i.e. $-0.97 \pm 0.27 b$ [66]. There is no experimental data to compare our result for the quadrupole moment of the first $3^{-}$state. We can, however, test the consistency of the fit by taking its opposite sign in the coupled-channels calculations. Fig. 9 shows the sensitivity of the fusion cross section and the fusion barrier distribution to the sign of the quadrupole moment of the first $3^{-}$state. The solid line corresponds to the optimal choice for the sign of the first $3^{-}$state, while the dotted line is obtained by inverting it. The use of the incorrect sign of the quadrupole moment destroys the good fit to the experimental data. Our calculations thus strongly suggest that the heavy-ion fusion reactions at low energies can provide an alternative method to determine the sign as well as the magnitude of the quadrupole moments of the low-lying excited states in spherical nuclei.

\section{SUMMARY AND CONCLUDING REMARKS}

We discussed the effects of anharmonic phonon excitations on heavy-ion fusion reactions at subbarrier energies and showed that they play an important role. We showed that the vibrational limit of the interacting boson model provides a useful framework to address these questions. There are mainly three effects of anharmonicities; the anharmonicity in the excitation energy spectra, the reorientation effects, and the finite boson number effects in the transition strength. We showed that the anharmonic effects in the energy spectra play only a minor role and the main effects come from the deviation of transition probabilities from the harmonic limit, i.e. both the reorientation and the finite $N$ effects. We found that the fusion barrier distribution strongly depends on the sign of the quadrupole moment of excited states. Using these properties, we have analyzed the ${ }^{16} \mathrm{O}+{ }^{148} \mathrm{Sm}$ fusion reaction and discussed the anharmonic properties of the phonon excitations in ${ }^{148} \mathrm{Sm}$. It was found that the best fit to the experimental data requires a negative and a positive quadrupole moments for the first $2^{+}$and $3^{-}$states of ${ }^{148} \mathrm{Sm}$, respectively. Since the quadrupole moment of the first $3^{-}$state of ${ }^{144} \mathrm{Sm}$ has been found to be negative [46], it would be interesting to measure that of the nucleus in between, i.e. ${ }^{146} \mathrm{Sm}$.

We have shown that calculations in the harmonic limit provide only qualitative results and the realistic situations are much more complex due to anharmonicities. Since harmonic calculations suggested that a fusion barrier distribution is quite sensitive to the number of phonon states excited during the fusion, it has been hoped that subbarrier fusion reactions offer an alternative method to identify the existence of higher phonon states. Actually, some analyses, aiming at identifying multi-phonon states, have been carried out. It is, however, apparent from our results that care has to be taken in such analyses. Our calculations show that fusion barrier distribution converges more rapidly with the number of phonons when the anharmonic effects are present compared with the 
case in their absence. Harmonic calculations may thus underestimate the maximum number of phonons in vibrational nuclei. It is vital to take into account anharmonic effects in order to properly identify the maximum number of phonons.

\section{ACKNOWLEDGMENTS}

The authors thank J.R. Leigh, M. Dasgupta, and D.J. Hinde for useful discussions and comments. The work of K.H. was supported by the Japan Society for the Promotion of Science for Young Scientists. This work was also supported by the Grant-in-Aid for General Scientific Research, Contract No.08640380, Monbusho International Scientific Research Program: Joint Research, Contract No. 09044051, from the Japanese Ministry of Education, Science and Culture.

\section{References}

[1] M. Beckerman, Rep. Prog. Phys. 51, 1047 (1988).

[2] A.B. Balantekin and N. Takigawa, Rev. Mod. Phys., in press.

[3] H. Esbensen, Nucl. Phys. A352, 147 (1981).

[4] M.A. Nagarajan, A.B. Balantekin, and N. Takigawa, Phys. Rev. C34, 894(1986).

[5] K. Hagino, N. Takigawa, J.R. Bennett, and D.M. Brink, Phys. Rev. C51, 3190 (1995).

[6] N. Rowley, G.R. Satchler, and P.H. Stelson, Phys. Lett. B254, 25 (1991).

[7] K. Hagino, N. Takigawa, and A.B. Balantekin, Phys. Rev. C 56, 2104 (1997).

[8] M. Dasgupta, D.J. Hinde, J.R. Leigh, and K. Hagino, Nucl. Phys. A, in press.

[9] J.X. Wei, J.R. Leigh, D.C. Weisser, J.O. Newton, S. Elfström, J.P. Lestone, J.X. Chen, D.G. Popescu, and D.J. Hinde, Nucl. Instrum. Methods Phys. Res. A 306, 557 (1991).

[10] J.R. Leigh, M. Dasgupta, D.J. Hinde, J.C. Mein, C.R. Morton, R.C. Lemmom, J.P. Lestone, J.O. Newton, H. Timmers, J.X. Wei, and N. Rowley, Phys. Rev. C52, 3151 (1995).

[11] J.X. Wei, J.R. Leigh, D.J. Hinde, J.O. Newton, R.C. Lemmon, S. Elfstrom, J.X. Chen, and N. Rowley, Phys. Rev. Lett. 67, 3368 (1991).

[12] R.C. Lemmon, J.R. Leigh, J.X. Wei, C.R. Morton, D.J. Hinde, J.O. Newton, J.C. Mein, M. Dasgupta, and N. Rowley, Phys. Lett. B316, 32 (1993).

[13] J.R. Leigh, N. Rowley, R.C. Lemmon, D.J. Hinde, J.O. Newton, J.X. Wei, J.C. Mein, C.R. Morton, S. Kuyucak, and A.T. Kruppa, Phys. Rev. C47, R437 (1993). 
[14] J.D. Bierman, P. Chan, J.F. Liang, M.P. Kelly, A.A. Sonzogni, and R. Vandenbosch, Phys. Rev. Lett. 76, 1587(1996); Phys. Rev. C54, 3068 (1996).

[15] C.R. Morton, M. Dasgupta, D.J. Hinde, J.R. Leigh, R.C. Lemmon, J.P. Lestone, J.C. Mein, J.O. Newton, H. Timmers, N. Rowley, and A.T. Kruppa, Phys. Rev. Lett. 72, 4074 (1994).

[16] H. Timmers, L. Corradi, A.M. Stefanini, D. Ackermann, J.H. He, S. Beghini, G. Montagnoli, F. Scarlassara, G.F. Segato, and N. Rowley, Phys. Lett. B399, 35 (1997).

[17] A.M. Stefanini, D. Ackermann, L. Corradi, J.H. He, G. Montagnoli, S. Beghini, F. Scalassara, and G.F. Segato, Phys. Rev. C52, R1727 (1995).

[18] A.M. Stefanini, D. Ackermann, L. Corradi, D.R. Napoli, C. Petrache, P. Spolaore, P. Bednarczyk, H.Q. Zhang, S. Beghini, G. Montagnoli, L. Mueller, F. Scarlassara, G.F. Segato, F. Sorame, and N. Rowley, Phys. Rev. Lett. 74, 864 (1995).

[19] D.M. Brink, A.F.R. de Toledo Piza, and A.K. Kerman, Phys. Lett. B19, 413 (1965).

[20] T. Tamura and T. Udagawa, Phys. Rev. 150, 783 (1966).

[21] A. Bohr and B. Mottelson, Nuclear Structure (Benjamin, New York, 1975), vol. 2.

[22] A. Aprahamian, D.S. Brenner, R.F. Casten, R.L. Gill, and A. Piotrowski, Phys. Rev. Lett. 59, 535 (1987).

[23] R.F. Casten, N.V. Zamfir, and D.S. Brenner, Phys. Rev. Lett. 71, 227 (1993).

[24] N.V. Zamfir and R.F. Casten, Phys. Lett. B341, 1 (1994).

[25] J. Kern, P.E. Garrett, J. Jolie, and H. Lehmann, Nucl. Phys. A593, 21 (1995).

[26] J. Kern and J. Jolie, Phys. Lett. B364, 207 (1995).

[27] K.-H. Kim, T. Otsuka, A. Gelberg, P. von Brentano, and P. Van Isacker, Phys. Rev. Lett. 76, 3514 (1996).

[28] C. Volpe, F. Catara, Ph. Chomaz, M.V. Andrés, and E.G. Lanza, Nucl. Phys. A589, 521 (1995).

[29] E.G. Lanza, M.V. Andrés, F. Catara, Ph. Chomaz, and C. Volpe, Nucl. Phys. A613, 445 (1997).

[30] G.F. Bertsch and H. Feldmeier, Phys. Rev. C56, 839 (1997).

[31] M.P. Metlay, J.L. Johnson, J.D. Canterbury, P.D. Cottle, C.W. Nestor Jr., S. Raman, and V.G. Zelevinsky, Phys. Rev. C52, 1801 (1995).

[32] P.D. Cottle and D.A. Bromley, Phys. Rev. C35, 1891 (1987). 
[33] I. Hamamoto, Nucl. Phys. A155, 362 (1970).

[34] B. Sorensen, Phys. Lett. 35B, 10 (1971).

[35] M. Guidetti, D.J. Rowe, and H. Chow, Nucl. Phys. A238, 225 (1975).

[36] A.M.R. Joye, A.M. Baxter, M.P. Fewell, D.C. Kean, and R.H. Spear, Phys. Rev. Lett. 38, 807 (1977).

[37] M. Yeh, P.E. Garrett, C.A. McGrath, S.W. Yates, and T. Belgya, Phys. Rev. Lett. 76, 1208 (1996).

[38] R.A. Gatenby, J.R. Vanhoy, E.M. Baum, E.L. Johnson, S.W. Yates, T. Belgya, B. Fazekas, Á. Veres, and G. Molnár, Phys. Rev. C41, R414 (1990).

[39] R.A. Gatenby, E.L. Johnson, E.M. Baum, S.W. Yates, D. Wang, J.R. Vanhoy, M.T. McEllistrem, T. Belgya, B. Fazekas, and G. Molnár, Nucl. Phys. A560, 633 (1993).

[40] M. Wilhelm, E. Radermacher, A. Zilges, and P. von Brentano, Phys. Rev. C 54, R449 (1996).

[41] E. Müller-Zanotti, R. Hertenberger, H. Kader, D. Hofer, G. Graw, Gh. Cata-Danil, G. Lazzari, and P.F. Bortingnon, Phys. Rev. C47, 2524 (1993).

[42] W. Urban, J.C. Bacelar, J. Jongman, W. Gast, G. Hebbinghaus, A. Krämer-Flecken, R.M. Lieder, M. Thoms, and O. Zell, Phys. Rev. C53, 2516 (1996).

[43] L. Bargioni, P.G. Bizzeti, A.M. Bizzeti-Sona, D. Bazzacco, S. Lunardi, P. Pavan, C. Rossi-Alvarez, G. de Angelis, G. Maron, and J. Rico, Phys. Rev. C51, R1057 (1995).

[44] M. Piiparinen, P. Kleinheinz, J. Blomqvist, A. Virtanen, A. Atac, D. Müller, J. Nyberg, T. Ramsoy, and G. Sletten, Phys. Rev. Lett. 70, 150 (1993).

[45] P. Kleinheinz, Prog. Part. Nucl. Phys. 28, 369 (1992).

[46] K. Hagino, N. Takigawa, and S. Kuyucak, Phys. Rev. Lett. 79, 2943 (1997).

[47] E. Cereda, M. Pignanelli, S. Micheletti, H.V. von Geramb, M.N. Harakeh, R. De Leo, G. D'Erasmo, and A. Pantaleo, Phys. Rev. C26, 1941 (1982).

[48] E.M. Takagui, G.R. Satchler, H. Takai, K. Koide, and O. Dietzsch, Nucl. Phys. A514, 120 (1990).

[49] M.J. Smithson, J.S. Lilley, M.A. Nagarajan, P.V. Drumm, R.A. Cunningham, B.R. Fulton, and I.J. Thompson, Nucl. Phys. A517, 193 (1990).

[50] F. Iachello and A. Arima, The Interacting Boson Model (Cambridge University Press, Cambridge, England, 1987).

[51] A.B. Balantekin, J.R. Bennett, and S. Kuyucak, Phys. Rev. C 48, 1269 (1993). 
[52] K. Hagino, N. Takigawa, M. Dasgupta, D.J. Hinde, and J.R. Leigh, Phys. Rev. C55, 276 (1997).

[53] N. Takigawa and K. Ikeda, in Proceedings of the Symposium on Many Facets of Heavy Ion Fusion Reactions, edited by W. Henning et al.(Argonne National Laboratory Report No. ANL-PHY-87-1), 1986, p.613.

[54] K. Hagino, N. Takigawa, A.B. Balantekin, and J.R. Bennett, Phys. Rev. C52, 286 (1995).

[55] A.T. Kruppa, P. Romain, M.A. Nagarajan, and N. Rowley, Nucl. Phys. A560, 845 (1993).

[56] R.F. Casten and D.D. Warner, Rev. Mod. Phys. 60, 389 (1988).

[57] J.N. Ginocchio, Nucl. Phys. A376, 438 (1982).

[58] A.B. Balantekin, J.R. Bennett, and S. Kuyucak, Phys. Rev. C49, 1079 (1994).

[59] A.B. Balantekin, J.R. Bennett, and S. Kuyucak, Phys. Rev. C49, 1294 (1994).

[60] A.F. Barfield, B.R. Barrett, J.L. Wood, and O. Scholten, Ann. Phys. (N.Y.) 182, 344 (1988).

[61] A.F. Barfield, P. von Brentano, A. Dewald, K.O. Zell, N.V. Zamfir, D. Bucurescu, M. Ivascu, and O. Scholten, Z. Phys. A332, 29 (1989).

[62] M. Pignanelli, N. Blasi, S. Micheletti, R. de Leo, M.A. Hofstee, J.M. Schippers, S.Y. van der Werf, and M.N. Harakeh, Nucl. Phys. A519, 567 (1990).

[63] A. Jungclaus, H.G. Börner, J. Jolie, S. Ulbig, R.F. Casten, N.V. Zamfir, P. von Brentano, and K.P. Lieb, Phys. Rev. C47, 1020 (1993).

[64] P.R. Christensen and A. Winther, Phys. Lett. B65, 19 (1976).

[65] K. Hagino, N. Takigawa, M. Dasgupta, D.J. Hinde, and J.R. Leigh, Phys. Rev. Lett. 79, 2014 (1997).

[66] L.K. Peker, Nucl. Data Sheets 59, 393 (1990).

[67] H. Esbensen and B.B. Back, Phys. Rev. C54, 3109 (1996).

[68] L.C. Vaz, J.M. Alexander, and G.R. Satchler, Phys. Rep. 69, 373 (1981).

[69] A. Wolf and R.F. Casten, Phys. Rev. C36, 851 (1987).

[70] O. Scholten, Phys. Lett. B127, 144 (1983). 


\section{Figure Captions}

Fig. 1: Schematic representation of the coupling scheme in vibrational models. The upper part is for the anharmonic vibrator, while the lower part for the harmonic oscillator. See text for the notation.

Fig. 2: Effects of anharmonicity of the quadrupole vibration on the excitation function of the fusion cross section (the upper panel) and fusion barrier distribution (the lower panel). The dotted line is the result in the harmonic limit, while the solid line takes the anharmonic effects of the vibrational excitations into account. The linear order coupling is assumed.

Fig. 3: Dependence of the fusion cross section (the upper panel) and fusion barrier distribution (the lower panel) on the deviation of the energy spectrum from the harmonic limit. The solid, the dotted, and the dashed lines are obtained by setting the parameter $\delta$ in Eq. (16) to be zero, 0.5 , and $-0.5 \mathrm{MeV}$, respectively. The dot-dashed line is the result in the degenerate limit in the anharmonic vibrator coupling. The phonon spectrum is truncated at the double phonon levels. The full order couplings are included.

Fig. 4: Dependence of the fusion cross section (the upper panel) and fusion barrier distribution (the lower panel) on the sign of the quadrupole moment of the first $2^{+}$state. The solid and the dotted lines correspond to the cases for the negative and the positive static quadrupole moments, respectively. The dashed line was obtained by setting the quadrupole moment of the excited states to be zero. The dot-dashed line is the result of the corresponding calculations in the harmonic limit.

Fig. 5: Convergence of the fusion cross section (the upper panel) and fusion barrier distribution (the lower panel) as functions of the number of phonon states included in the coupled-channels equations, which are indicated in the inset. The total boson number $N$ is fixed to be four in all cases.

Fig. 6: The same as Fig. 5, but for the harmonic oscillator coupling.

Fig. 7: Comparison of the experimental fusion barrier distribution with the results of coupled-channels calculations in the harmonic limit for the ${ }^{16} \mathrm{O}+{ }^{148} \mathrm{Sm}$ reaction. The dotted lines include the single octupole excitations, while the solid lines take the double octupole phonon couplings into account. The experimental data are taken from Ref. [10].

Fig. 8: Comparison of the experimental fusion cross section (the upper panel) and fusion barrier distribution (the lower panel) with the coupled-channels calculations for the ${ }^{16} \mathrm{O}$ $+{ }^{148} \mathrm{Sm}$ reaction. The experimental data are taken from Ref. [10]. The anharmonic effects in the quadrupole and the octupole vibrational excitations in ${ }^{148} \mathrm{Sm}$ are taken into account in the $s d f$ - IBM. The solid line is the result when the best fit parameters are used, while the dashed line is obtained by setting the quadrupole moments of all the excited 
states to be zero. The results including the four phonon couplings in the harmonic limit is denoted by the dotted line.

Fig. 9: Dependence of the fusion cross section (the upper panel) and fusion barrier distribution (the lower panel) on the sign of the static quadrupole moment of the first $3^{-}$state for the ${ }^{16} \mathrm{O}+{ }^{148} \mathrm{Sm}$ reaction. The solid and the dotted lines correspond to the cases where the quadrupole moment of the first 3 - state in ${ }^{148} \mathrm{Sm}$ is positive and negative, respectively. 


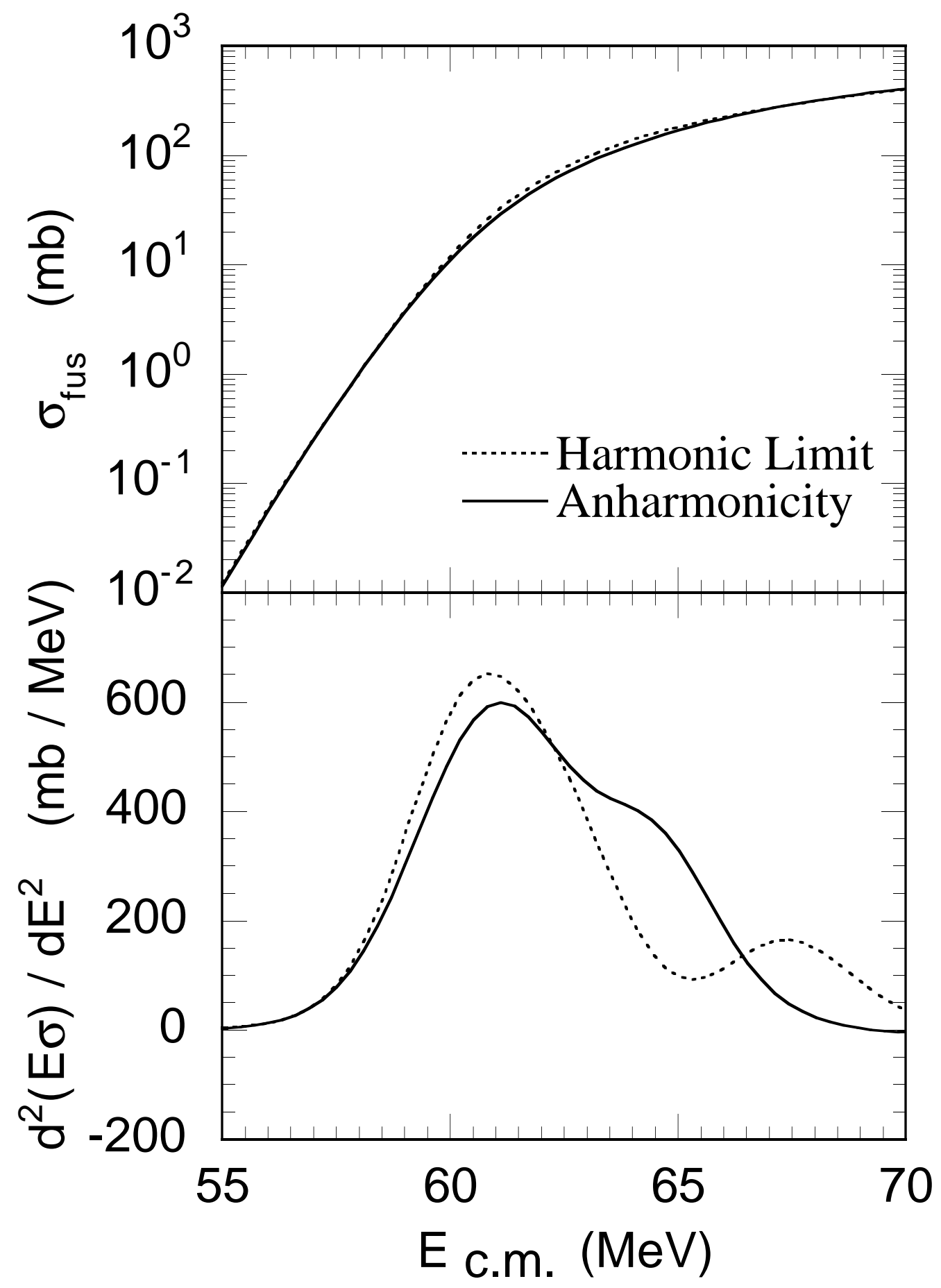




\section{$\underline{A H V}$}

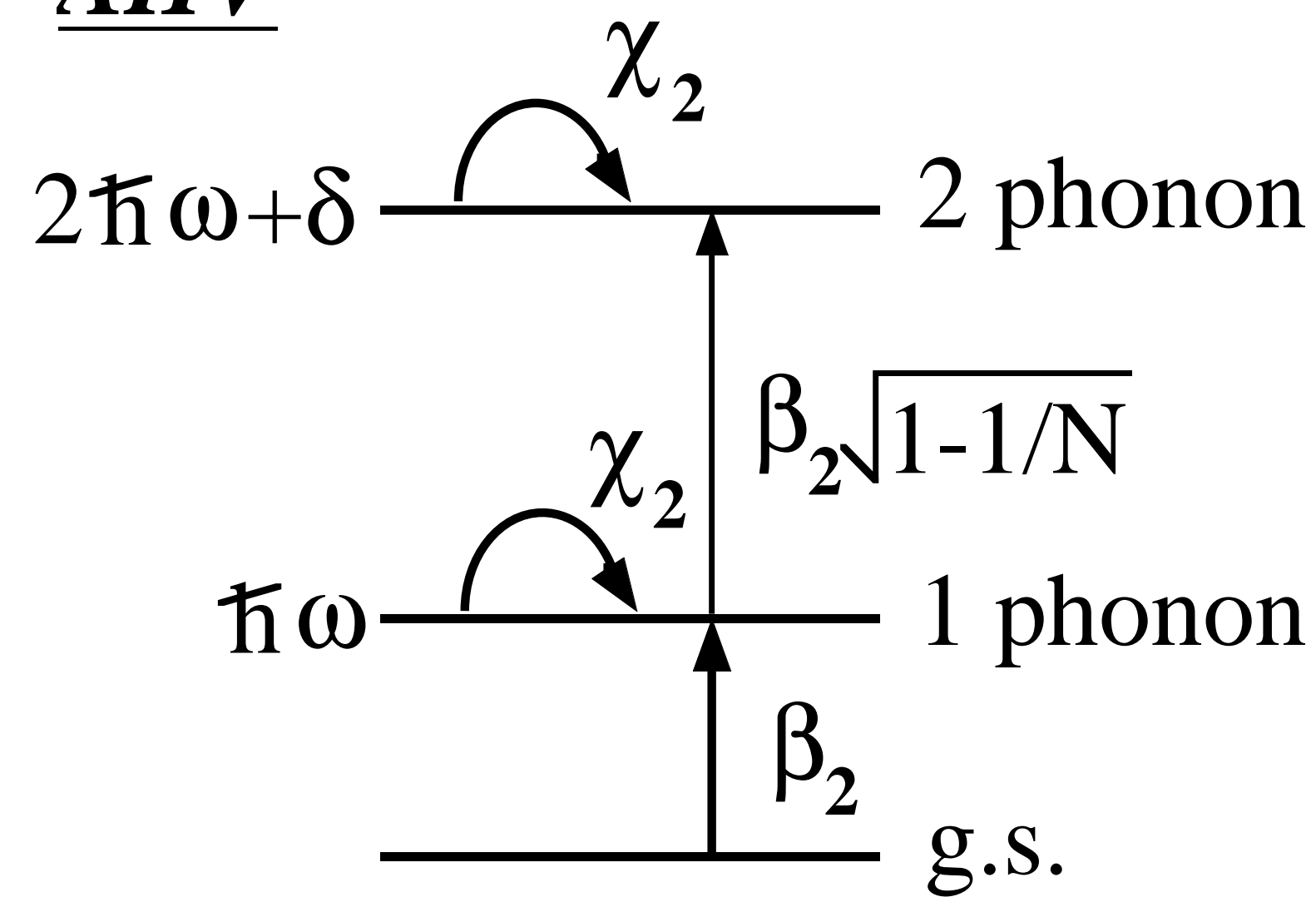

\section{$\underline{H O}$}

$2 \hbar \omega$

2 phonon

$\beta_{2}$

$\hbar \omega$

1 phonon

$\beta_{2}$

$$
\text { g.s. }
$$




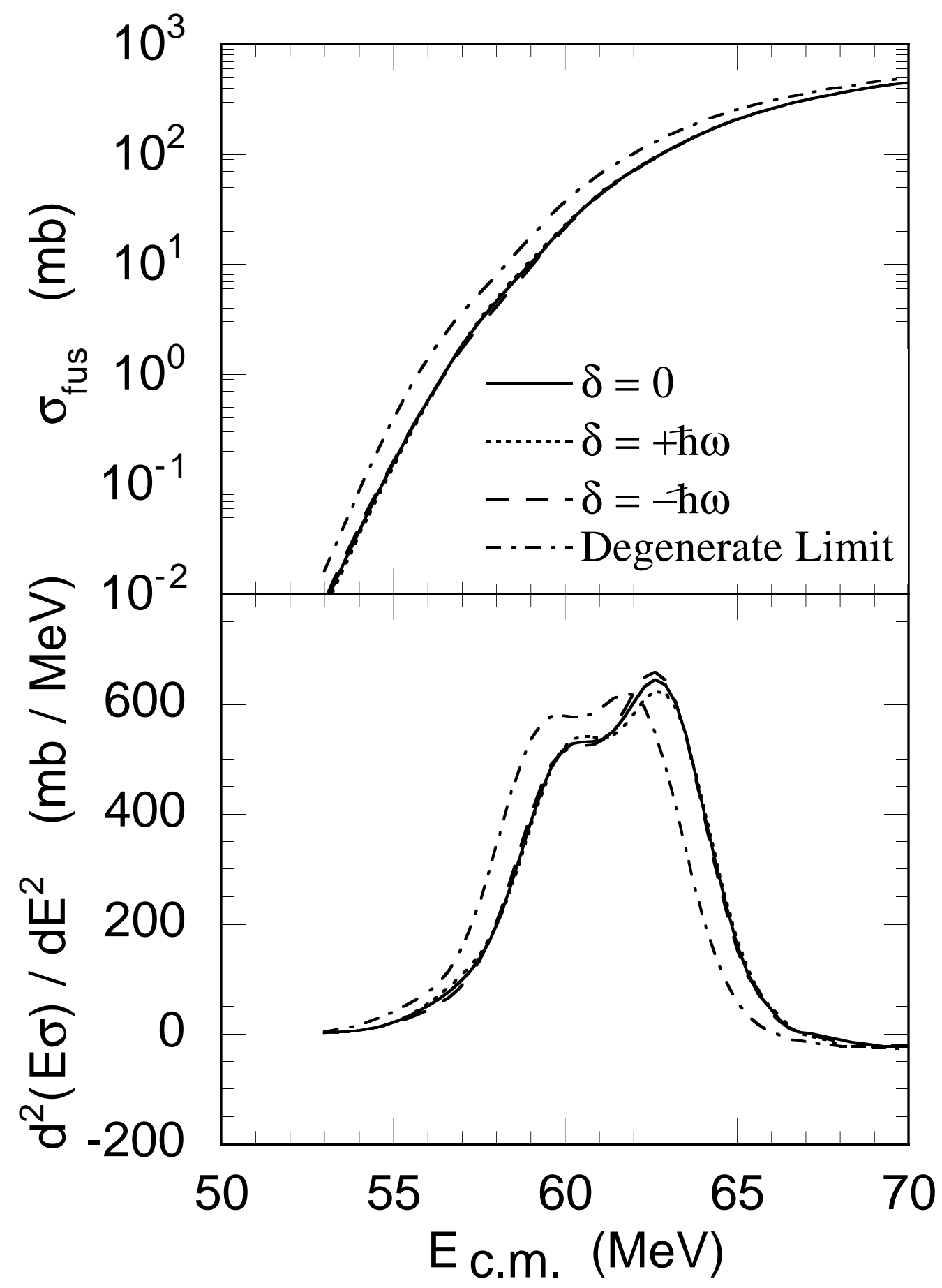




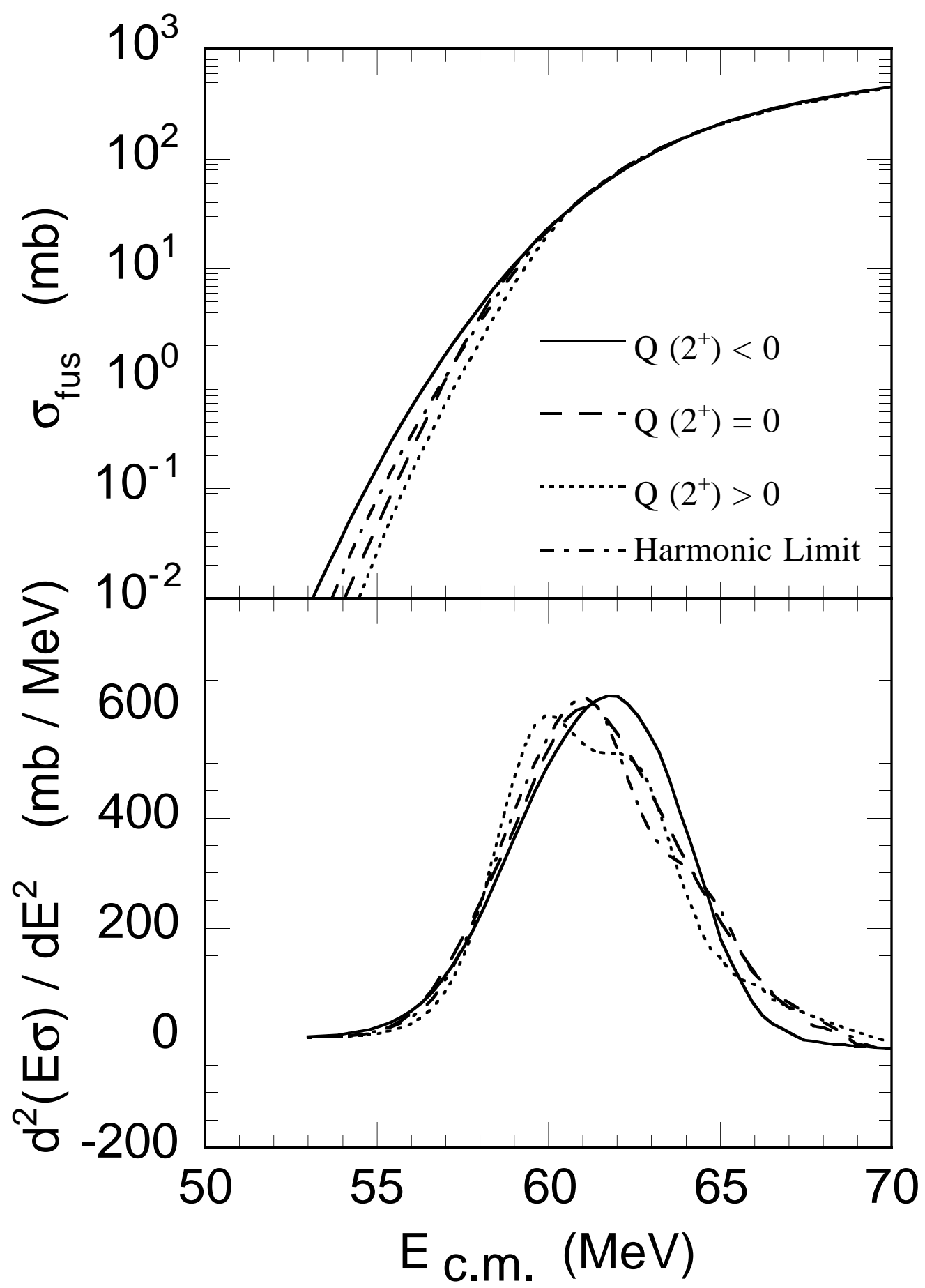




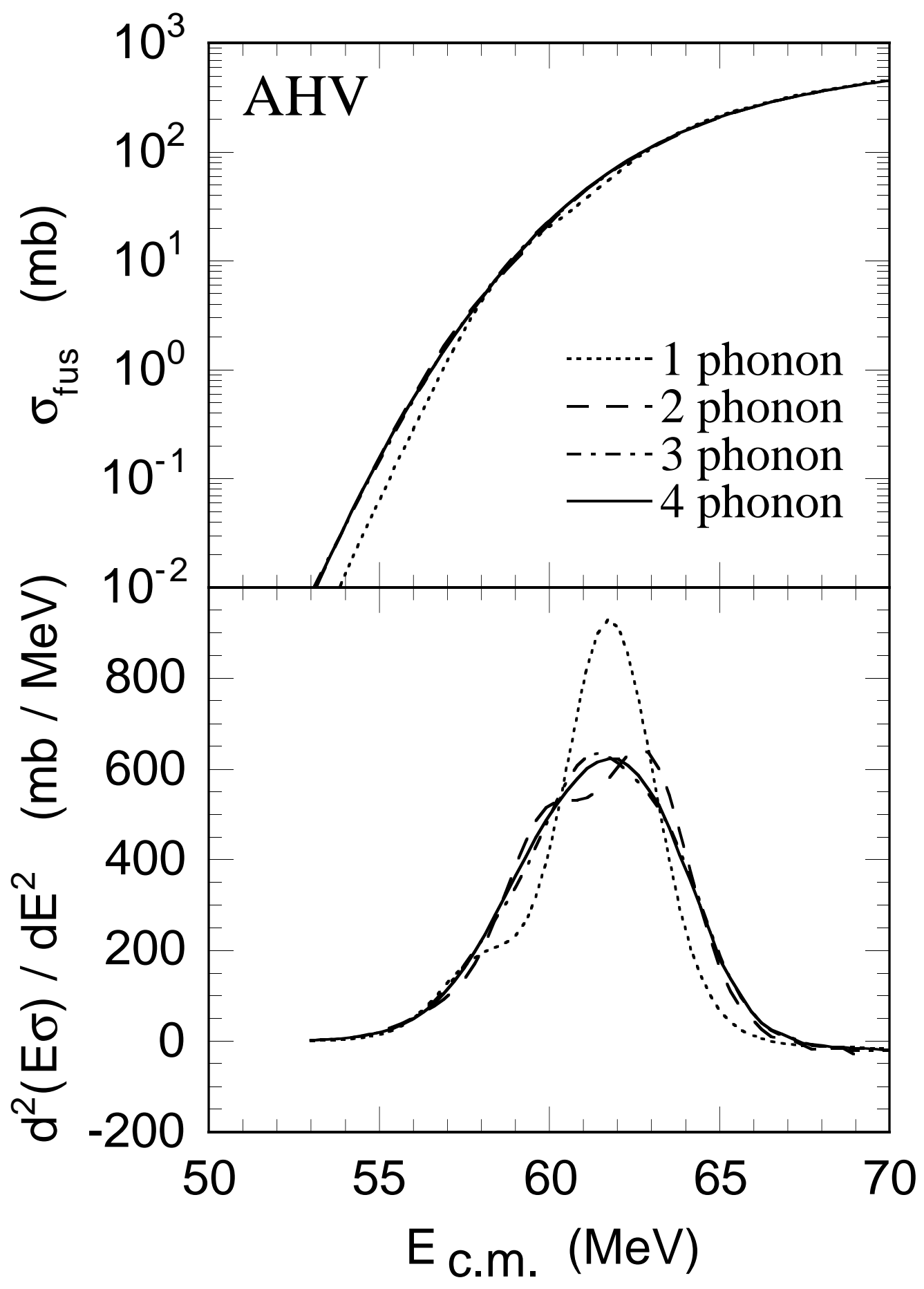




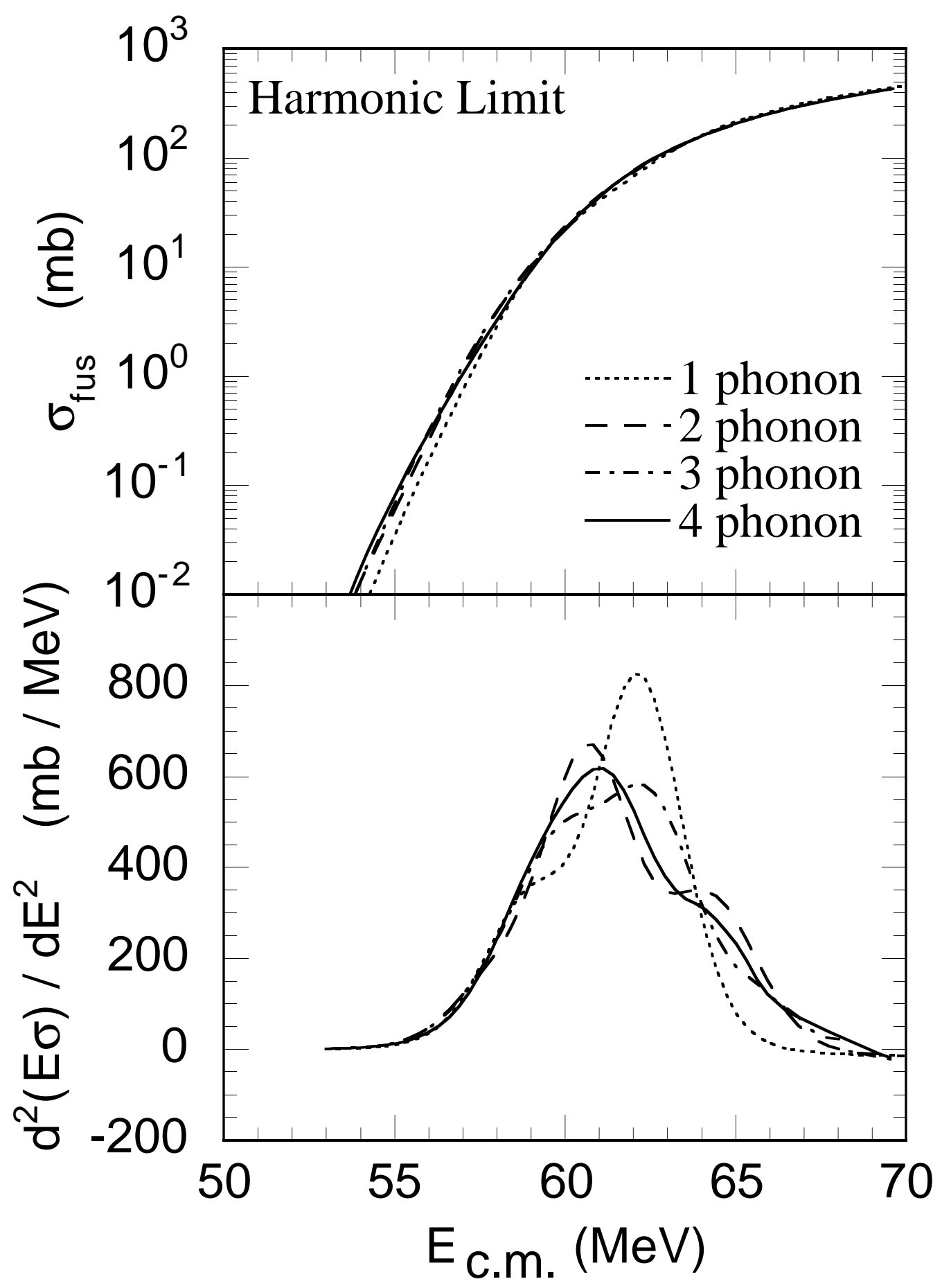




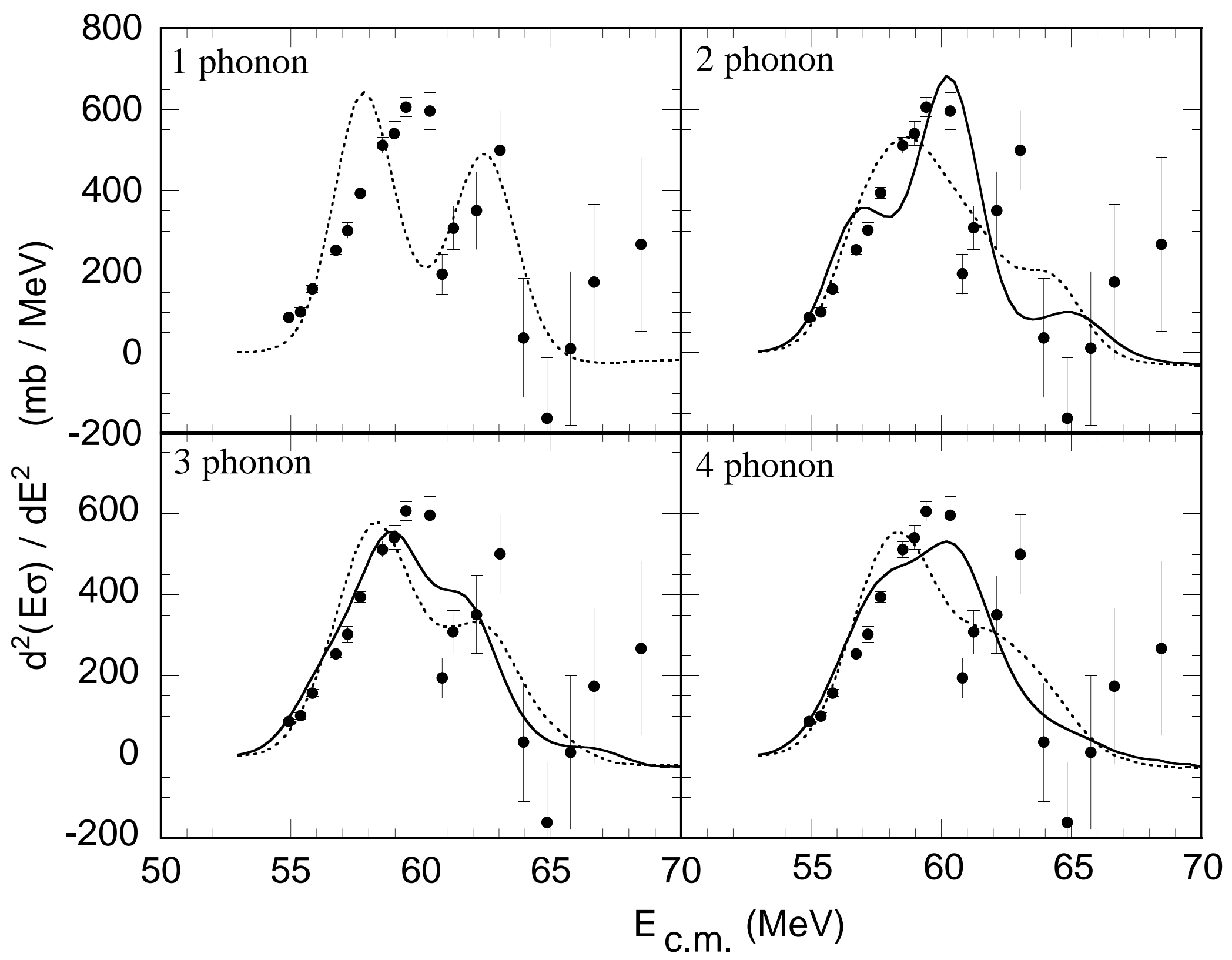




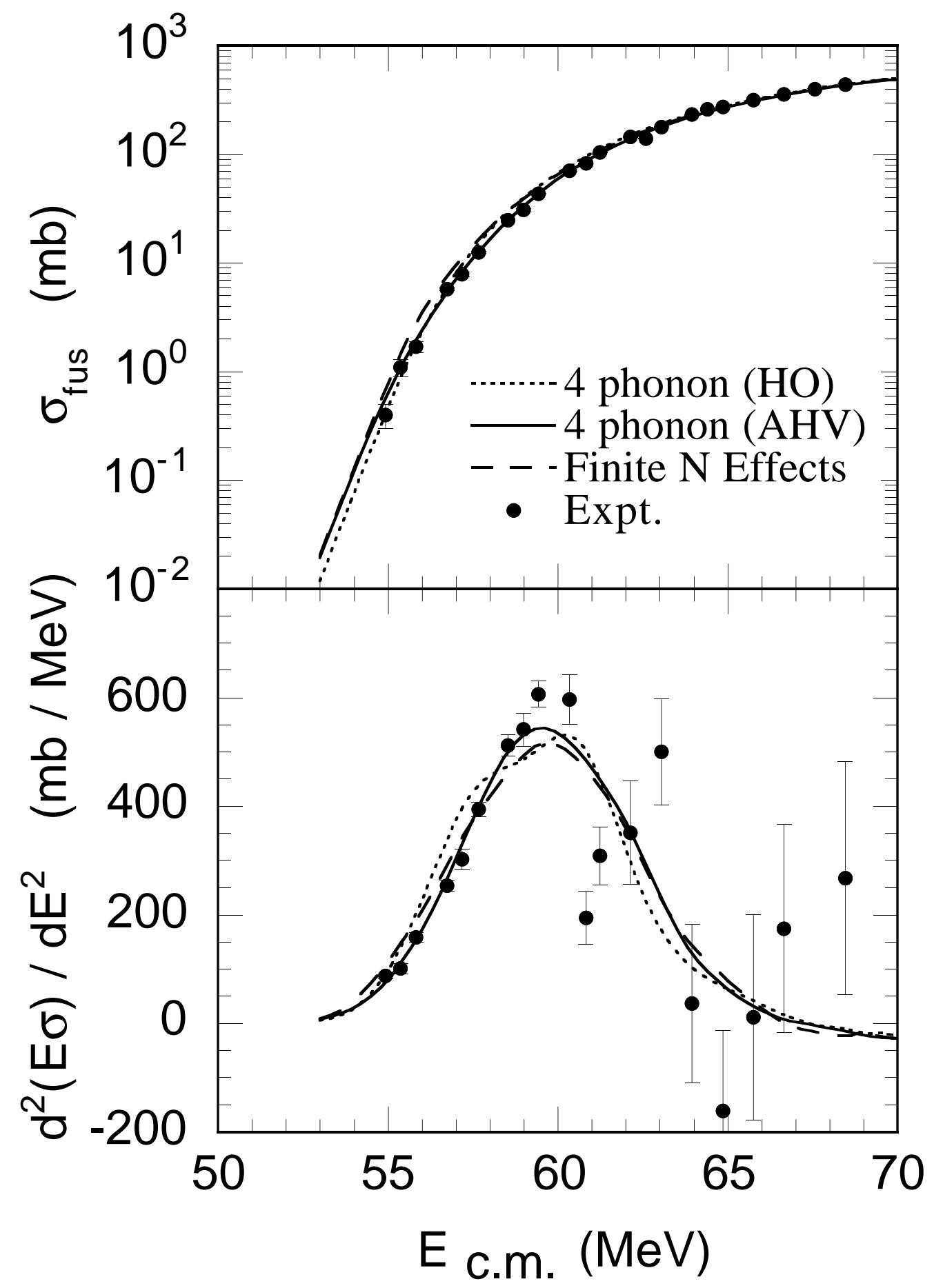




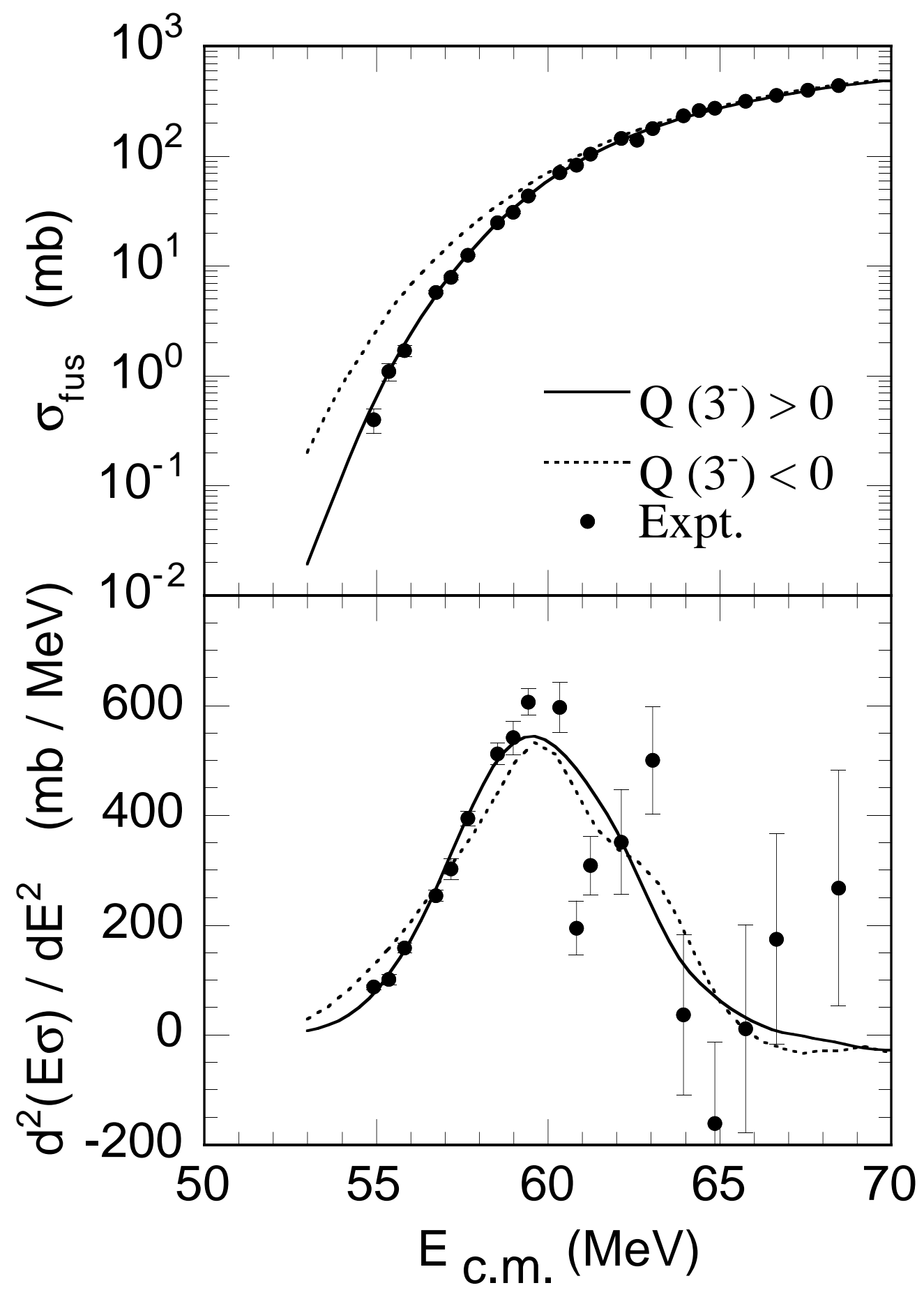

